\title{
The Effect of Particle Size on Pt/C Electrocatalyst Degradation in Proton Exchange Membrane Fuel Cells
}

\author{
D. J. Groom*, S. Rajasekhara *, S. A. Matyas*, Z. Yang**, M. Gummalla**, S. C. Ball***, P. J. \\ Ferreira* \\ * Materials Science \& Engineering Program, The University of Texas - Austin, Austin, TX - 78712 \\ ** United Technology Research Center, East Hartford, CT 06108 \\ *** Johnson Matthey Technology Center, Blount's Court, Sonning Common, Reading, RG4 9NH, \\ UK
}

Pt nanoparticle electrocatalysts in Proton Exchange Membrane Fuel Cells (PEMFCs) have been shown to undergo irreversible degradation through coalescence and Pt dissolution, the degree of which vary with initial particle size. Such degradation leads to losses in the performance and operating hours of the PEMFC [1]. In this work, we study the relationship between Pt nanocatalyst size and degradation mechanisms for PEMFC cathodes cycled between 0.6 and $1.0 \mathrm{~V}$ in triangular wave with $32 \mathrm{~s}$ time period. Furthermore, to address the nanoparticle morphology, we use for the first time roundness and circularity parameters as novel metrics.

Two cycled membrane electrode assemblies (MEAs), S1 and S2, whose cathodes were initially loaded with Pt nanoparticles of sizes $2 \mathrm{~nm}$ and $12 \mathrm{~nm}$, respectively were microtomed for crosssection transmission electron microscopy (TEM) analysis. Subsequently, TEM images from three separate regions of the cathode, $3 \mu \mathrm{m}$ in length, thereby covering the approximate $10 \mu \mathrm{m}$ electrode thickness (Fig. 1) were acquired in a JEOL 2010F operated at $200 \mathrm{kV}$ and analyzed.

In addition to individual spherical particles, the TEM observations identified nanoparticles with two distinct morphologies: (i) coalesced (Figs. 2a and 3a) and (ii) dendritic (Figs. 2b and 3b). As expected, coalesced and dendritic morphologies were more prevalent in sample $\mathrm{S} 1$ because smaller nanocatalysts have higher dissolution and mobility rates [1]. For example in region A, the frequency of coalesced nanoparticles was found to be $9.0 \%$ for sample S1 and $4.5 \%$ for sample S2, while the dendritic particles were $3.5 \%$ for sample S1 and $2.5 \%$ for sample S2. However, sample S1 exhibits a more pronounced dissolution than what is suggested by the dendritic frequency, as observed by the dense agglomeration of $\mathrm{Pt}$ nanoparticles along the cathode membrane interface, which was completely absent in the sample S2.

The two morphologies were subsequently defined by a ratio $\mathrm{R}$ of roundness to circularity, for which $\mathrm{R}>1$ for dendritic particles and $\mathrm{R}<1$ for coalesced particles. The ratio $\mathrm{R}$ provides a means to quantify the severity and the mechanism of degradation for a particular nanoparticle. Roundness is more sensitive to elongation caused by coalesced particles along a central axis. Circularity is more sensitive to sharp discontinuities caused by variations in concavity and convexity, such as branching.

The presence of these morphologies provides insight into specific degradation mechanisms. In particular, the dendritic particles arise from the dissolution and redeposition of Pt, whereas the coalesced particles are the result of nanoparticles migrating and coalescing on the carbon support [2].

References

[1] Y-S. Horn et al., Top. Catal. 46 (2007) 285

[2] P. J. Ferreira et al., J. Electrochem. Soc. 152 (2005) A2256 


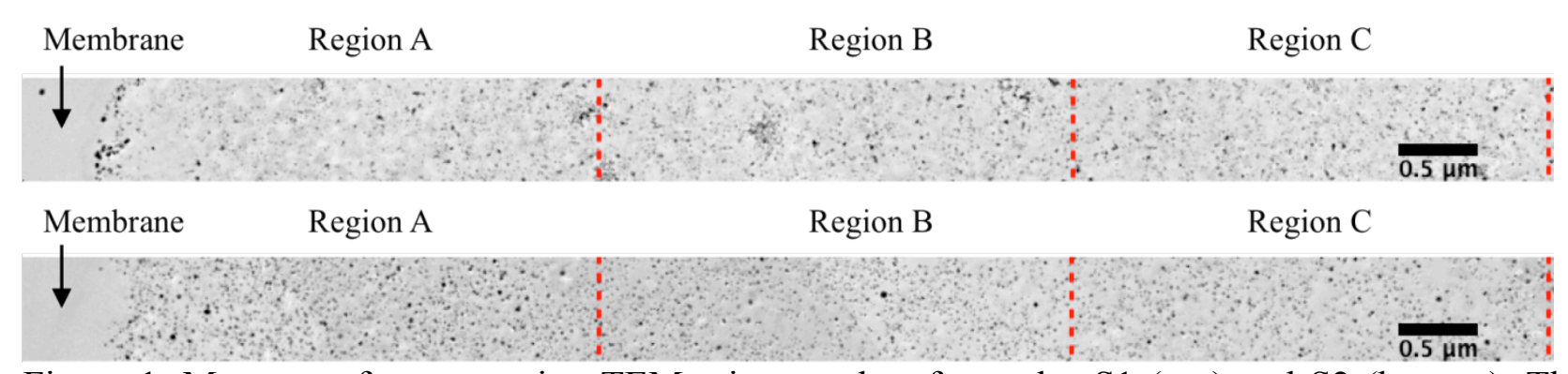

Figure 1. Montage of cross-section TEM micrographs of samples S1 (top) and S2 (bottom). The dashed red lines delineate regions $\mathrm{A}, \mathrm{B}$, and $\mathrm{C}$ of the cathode, which are measured $0-3,3-6$, and 6-9 $\mu \mathrm{m}$ away from the cathode membrane interface, respectively.
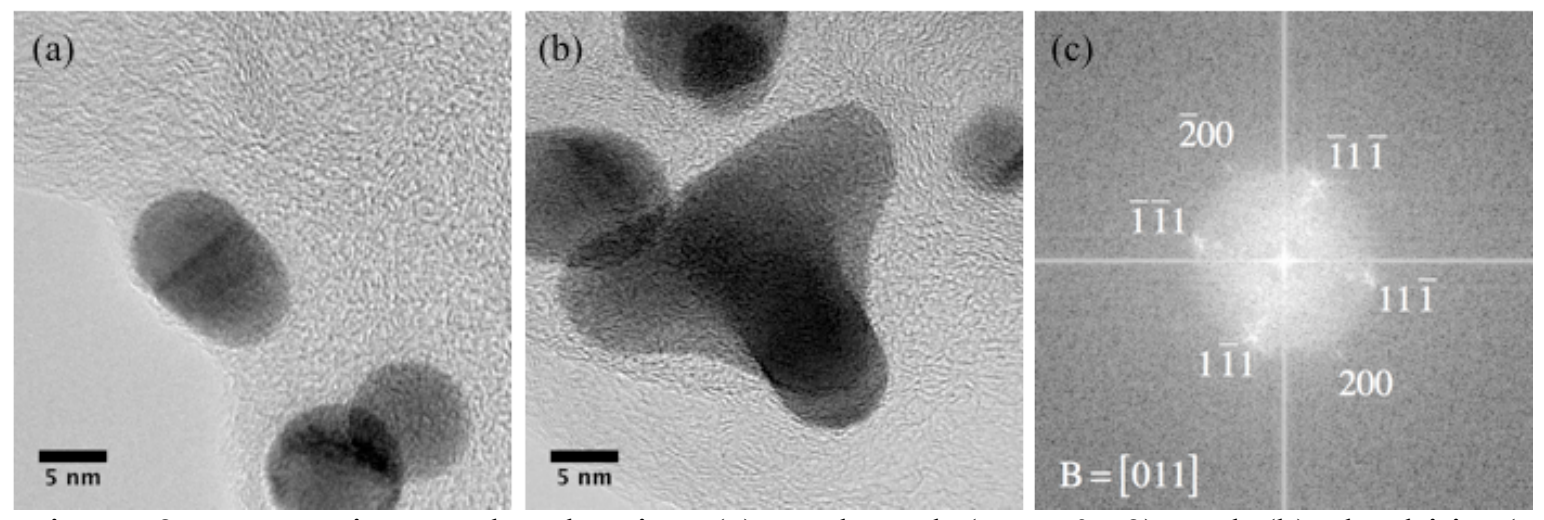

Figure 2. TEM micrographs showing (a) coalesced $(\mathrm{R}=0.79)$ and $(\mathrm{b})$ dendritic $(\mathrm{R}=1.35)$ nanoparticles located near the cathode membrane-interface of sample S1. (c) The Fast Fourier Transform of the dendritic particle in (b) indicates that it is a single crystal.
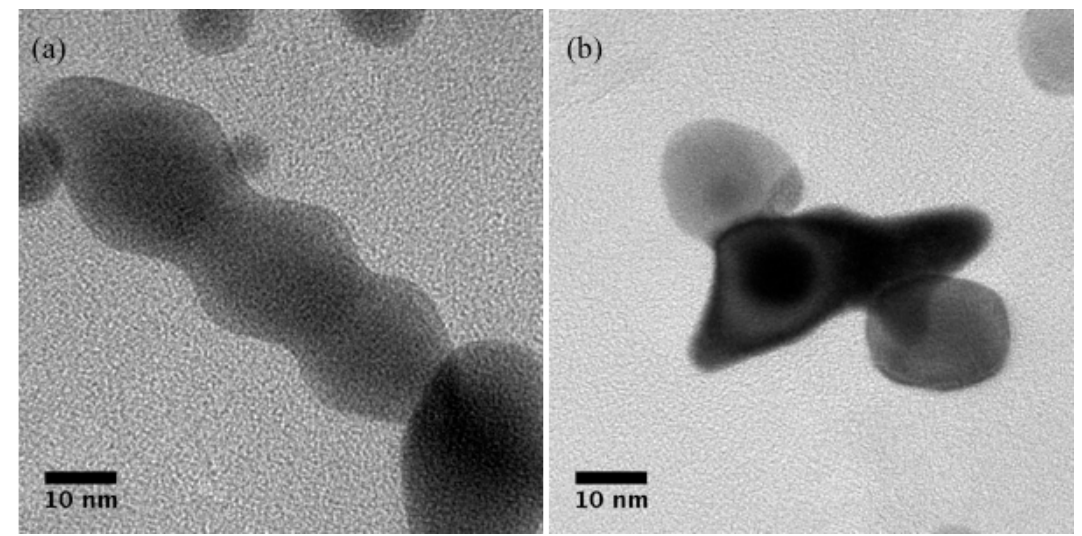

Figure 3. TEM micrographs showing $(\mathrm{a})$ coalesced $(\mathrm{R}=0.58)$ and $(\mathrm{b})$ dendritic $(\mathrm{R}=1.40)$ nanoparticles located near the cathode membrane interface of sample $\mathrm{S} 2$. 